\title{
Characterization of a photon counting test bed for space to ground optical pulse position modulation communications links
}

Jennifer M. Nappier, Brian E. Vyhnalek, Sarah A. Tedder, and Nicholas C. Lantz

NASA Glenn Research Center

Cleveland, Ohio

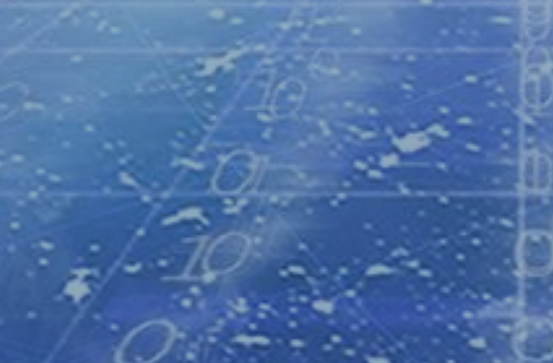




\section{Introduction}

\section{Motivation:}

- Real time photon counting optical ground receivers are needed to enable space to ground communications for both public and private applications.

- Future NASA photon counting optical communications missions: LCRD, 020, Psyche

\section{Strategy:}

1. Develop an optical communications photon counting test bed to enable development of a real time optical receiver which includes the following subsystems:

- Aft optics (photonic lantern), single photon counting detectors, and real time FPGA-based receiver.

2. Model key optical communications system parameters to understand impact on system performance

\section{Objective:}

- Utilize system test bed and model to predict system performance

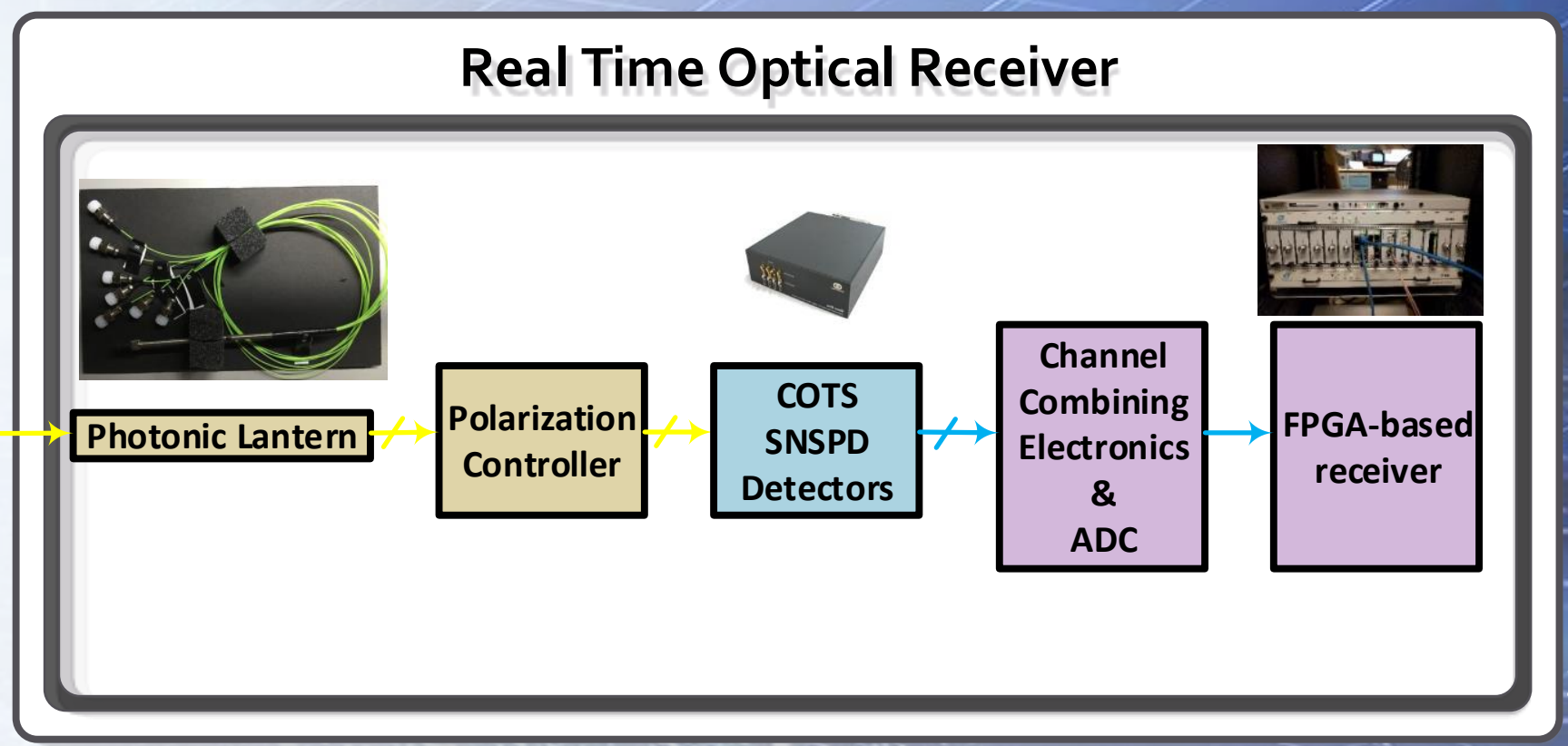




\section{Optical Communications System Test Bed}
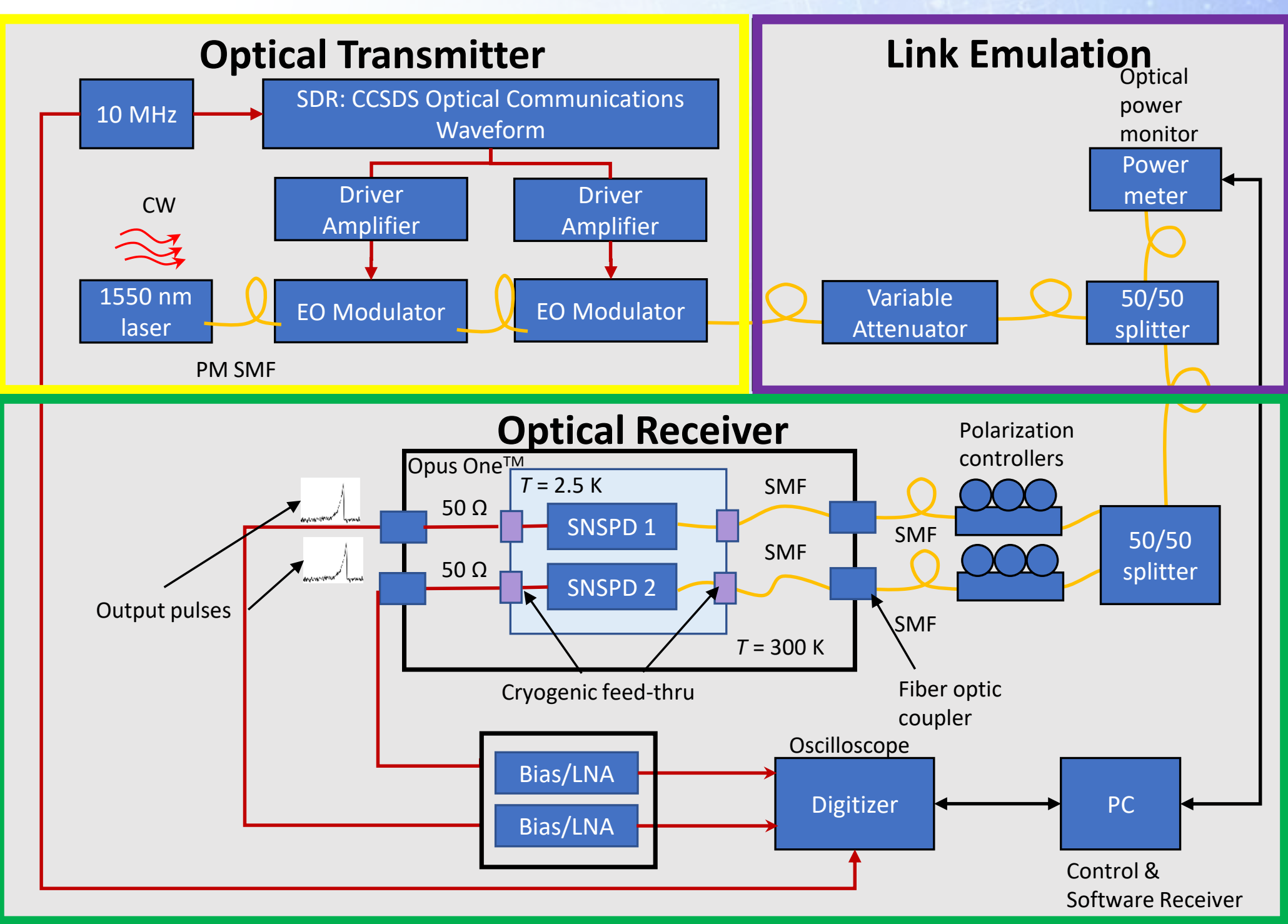


\section{Optical Transmitter - Software Defined Radio}

- Based on Harris Corporation Reconfigurable Space processor development card

- A custom optical mezzanine card performs serialization of electrical signal generated on FPGA

- Xilinx Virtex 7 FPGA houses the optical transmit waveform

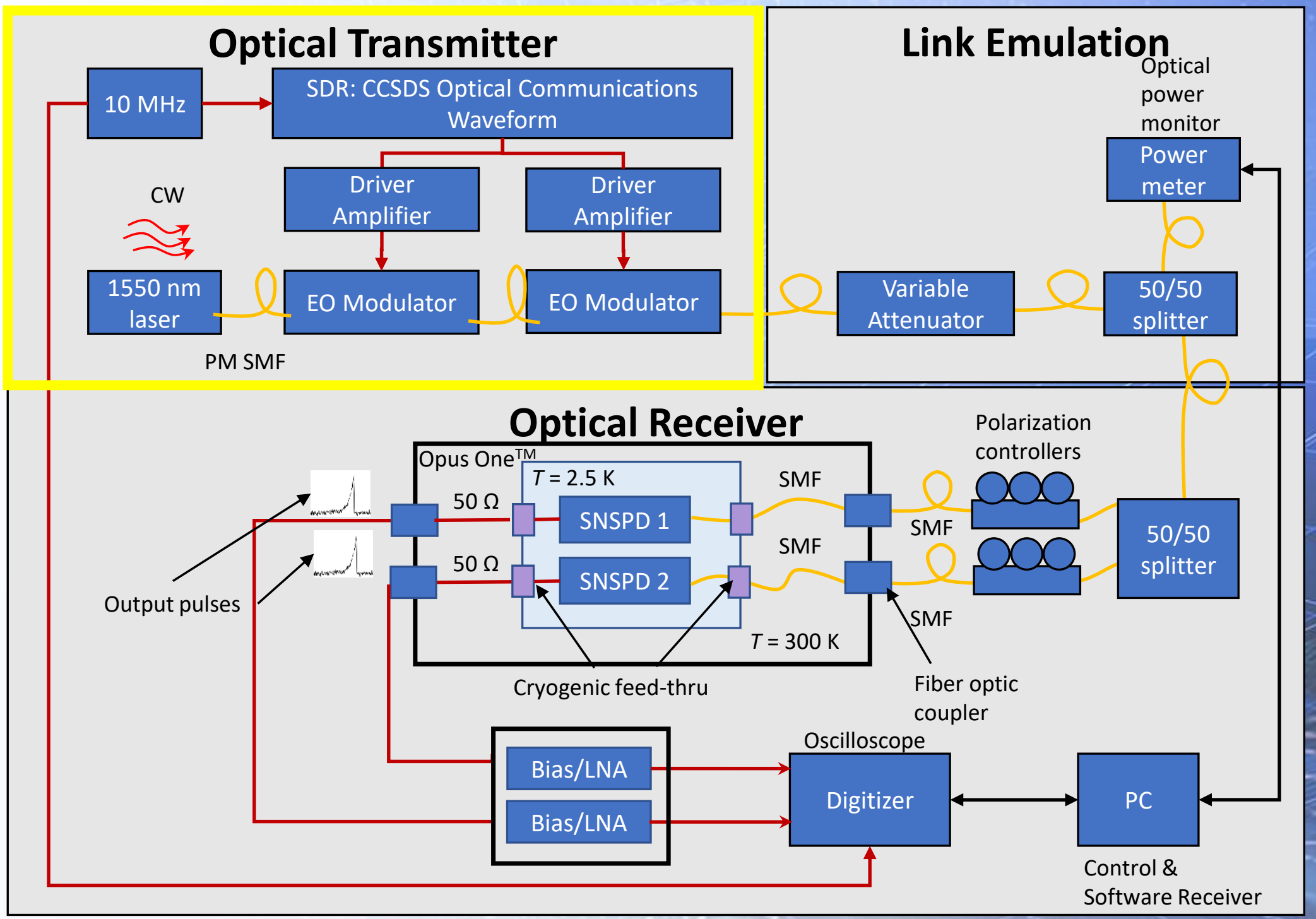




\section{Optical Transmitter -Waveform}

- Implements the full CCSDS Optical Communications Coding and Synchronization Red Book telemetry link

- Testing performed with the following waveform:

- PPM-32

- Code rate $1 / 3$

- Slot width $1 \mathrm{~ns}$

- Guard band: 8 slots (25\%)

- Data rate 40 Mbps

- Note: channel interleaver bypassed for testing purposes

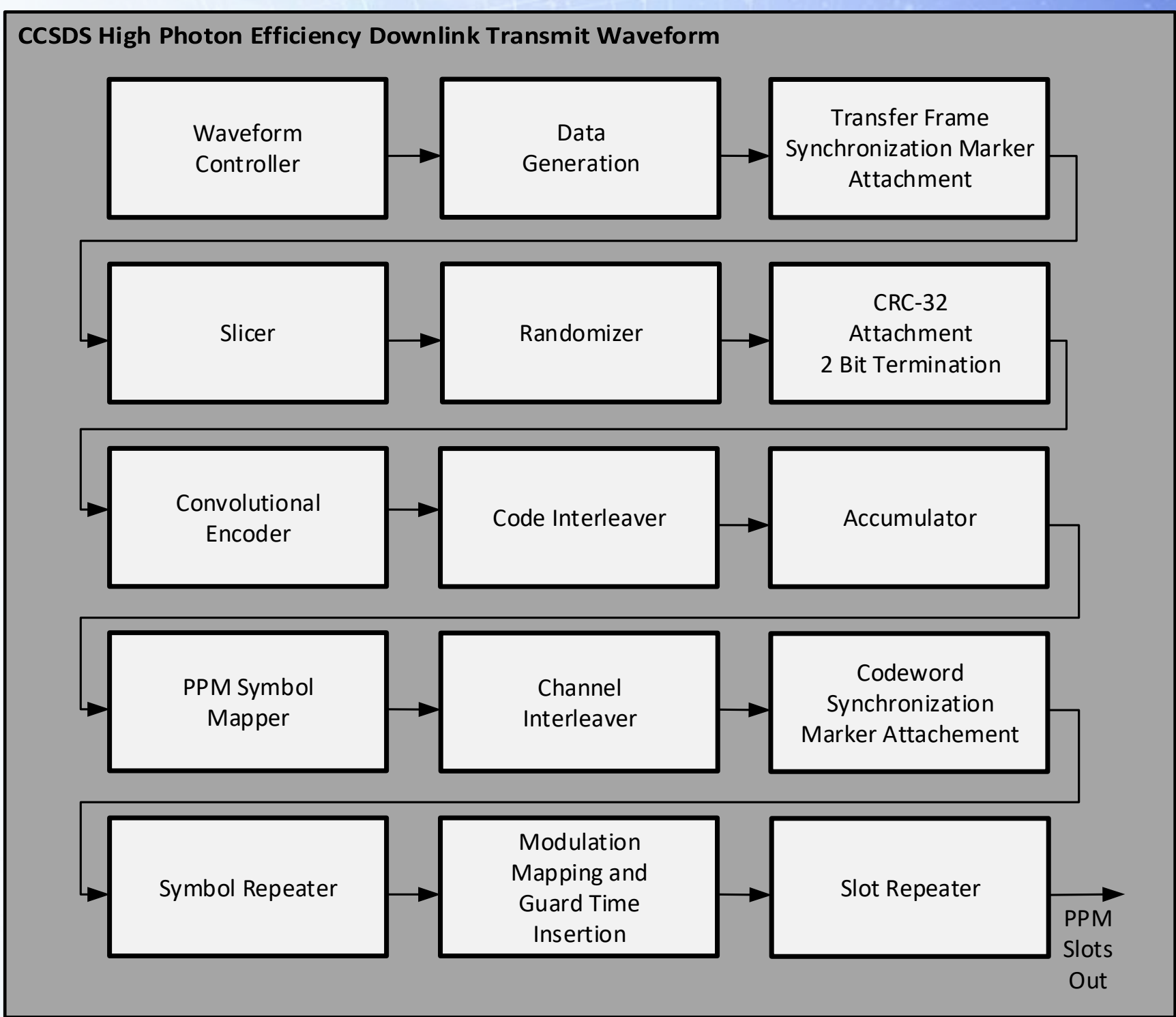




\section{Optical Transmitter - Electro-optic Modulator System ${ }^{1}$}

- Consists of two high extinction ratio electro-optic modulators cascaded in series

- Electrical signal driving modulators is offset in time to narrow the optical pulse position modulation signal, improving the extinction ratio

1. Lantz, N., Nappier, J., Vyhnalek, B. and Tedder, S. "Optical software defined radio transmitter extinction ratio enhancement with differential pulse carving," Proc. SPIE Free-Space Laser Communication and Atmospheric Propagation XXXI 10910 (62) 2019.

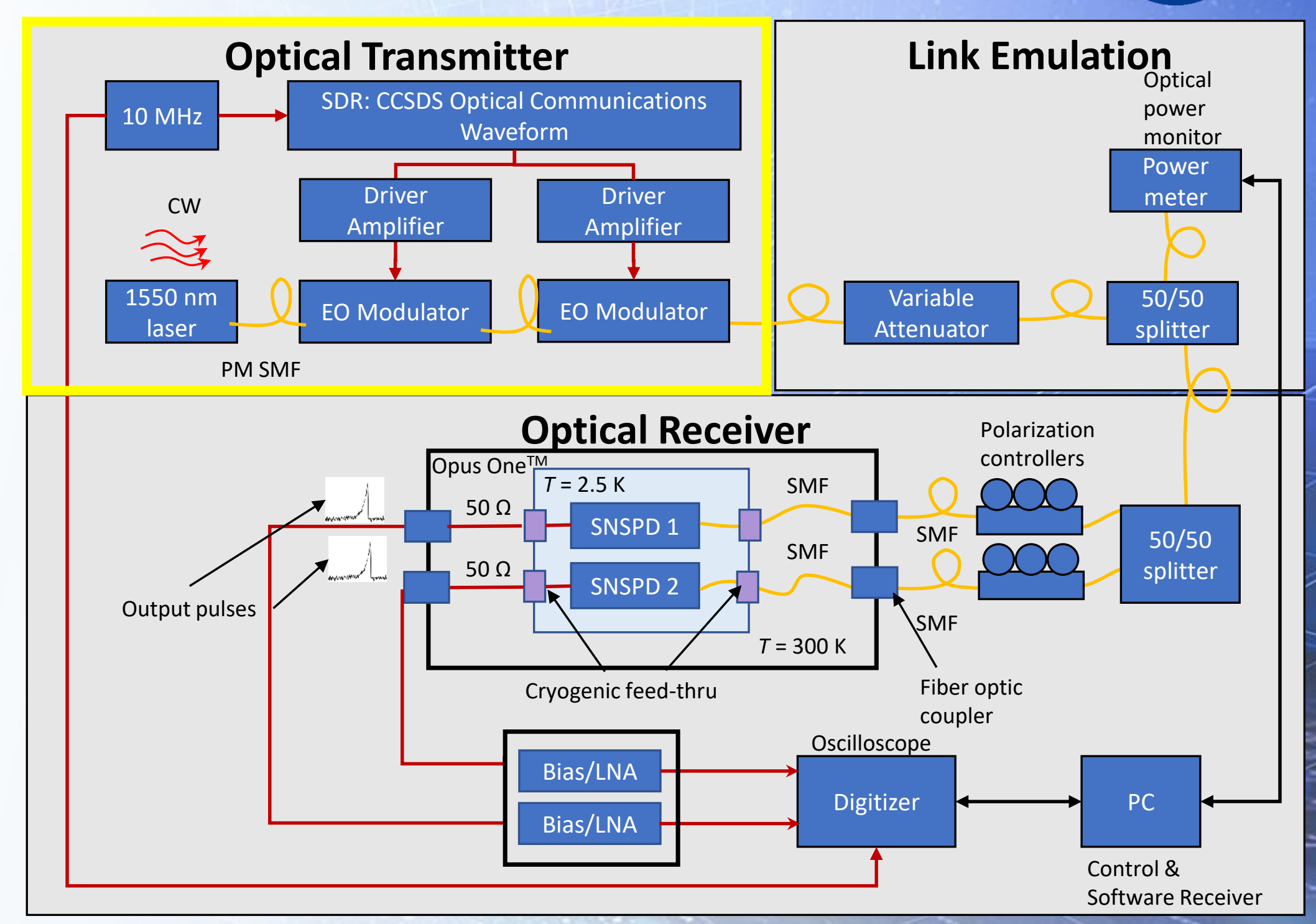

SPIE Photonics West 2019 


\section{Link Emulation}

- Free space loss is emulated with a variable attenuator

- No additional noise inserted

- $\mathrm{Kb} \approx 0.0001$ background photons/slot

- Power meter used to measure optical power into the receiver
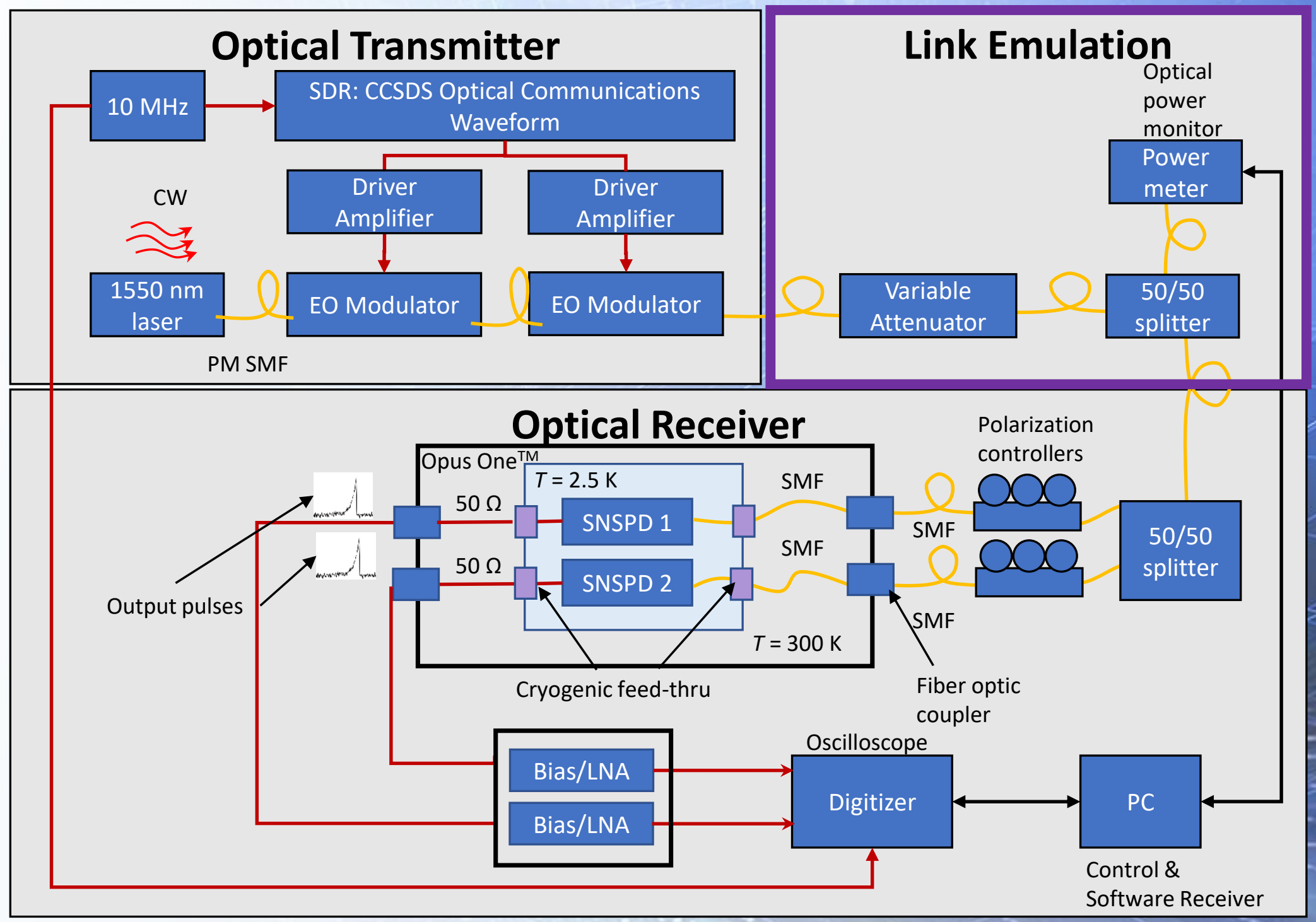


\section{Optical Receiver - Single Photon Counting Detectors ${ }^{2}$}

\section{Description:}

- Contains two single mode fiber coupled detectors from the Quantum Opus, Opus One ${ }^{\mathrm{TM}}$ system

\section{Characterization Results:}

- Detector pulse rise time

- 850 ps

- Detector reset time

- 18-20 ps

- Maximum detection efficiency (polarization dependent)

- $\quad 80 \%$ at maximum point

- $\quad 50-60 \%$ at operating point due to detector blocking losses (1.5 dB blocking loss)

- Detector jitter full width half max:

- Channel 1:68 ps

- Channel 2: 85 ps

2. Vyhnalek, B., Tedder, S., and Nappier, J. "Few-mode fiber coupled superconducting nanowire single-photon detectors for photon efficient optical communications," Proc. SPIE Free-Space Laser Communication and Atmospheric Propagation XXXI 10910 (11) (2019).
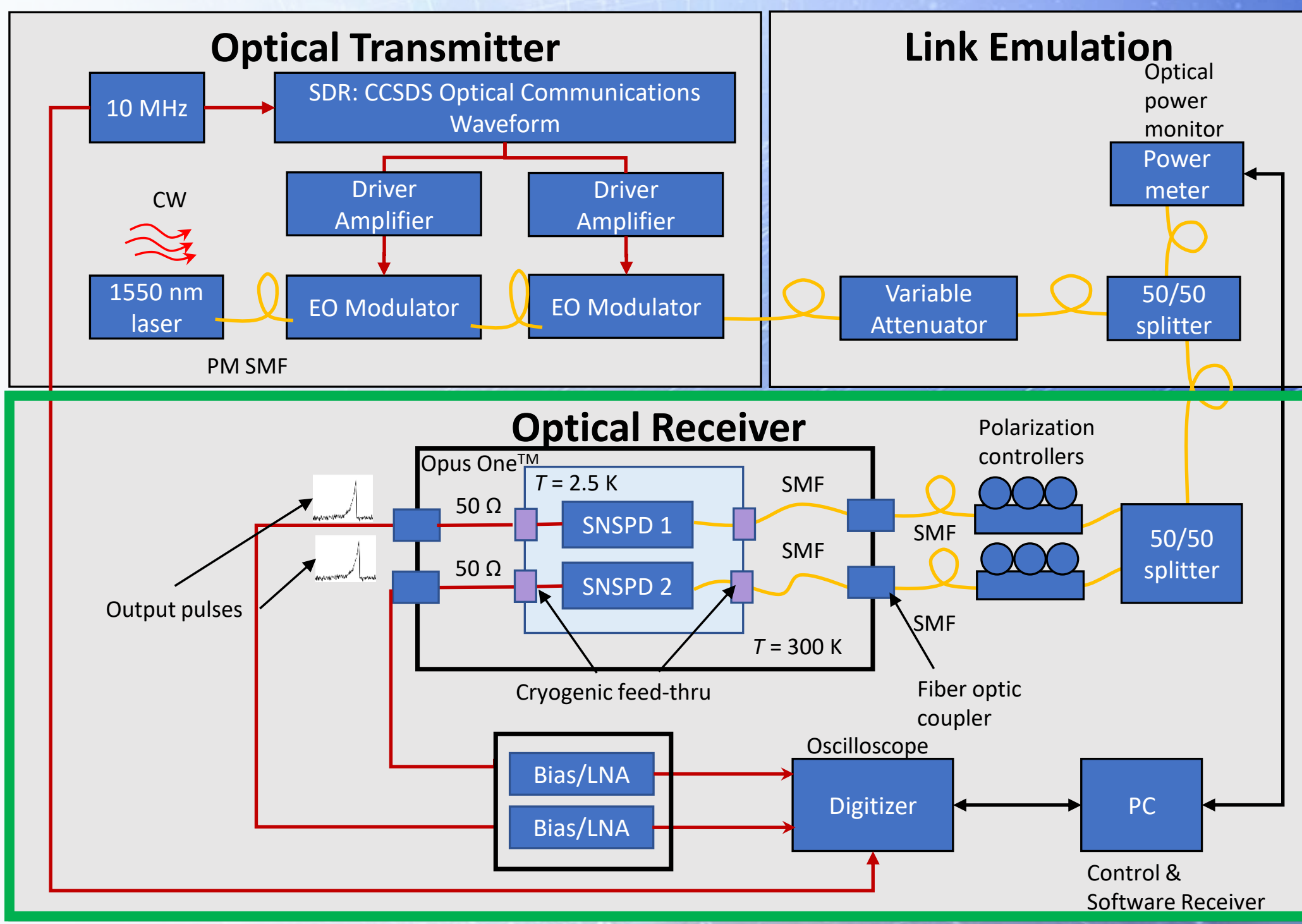


\section{Optical Receiver - Waveform}

- Detector pulses are sampled at $2 \mathrm{GHz}$ with an oscilloscope and post-processed using a Matlab receiver model

- SCPPM decoder performs iterative decoding using the BCJR algorithm

- Sample jitter introduced by 2 GHz sampling of 850 ps detector pulse is $\sim 45$ ps RMS

- Calculated total receiver jitter:

- Channel 1: 61 ps RMS

- Channel 2: 68 ps RMS

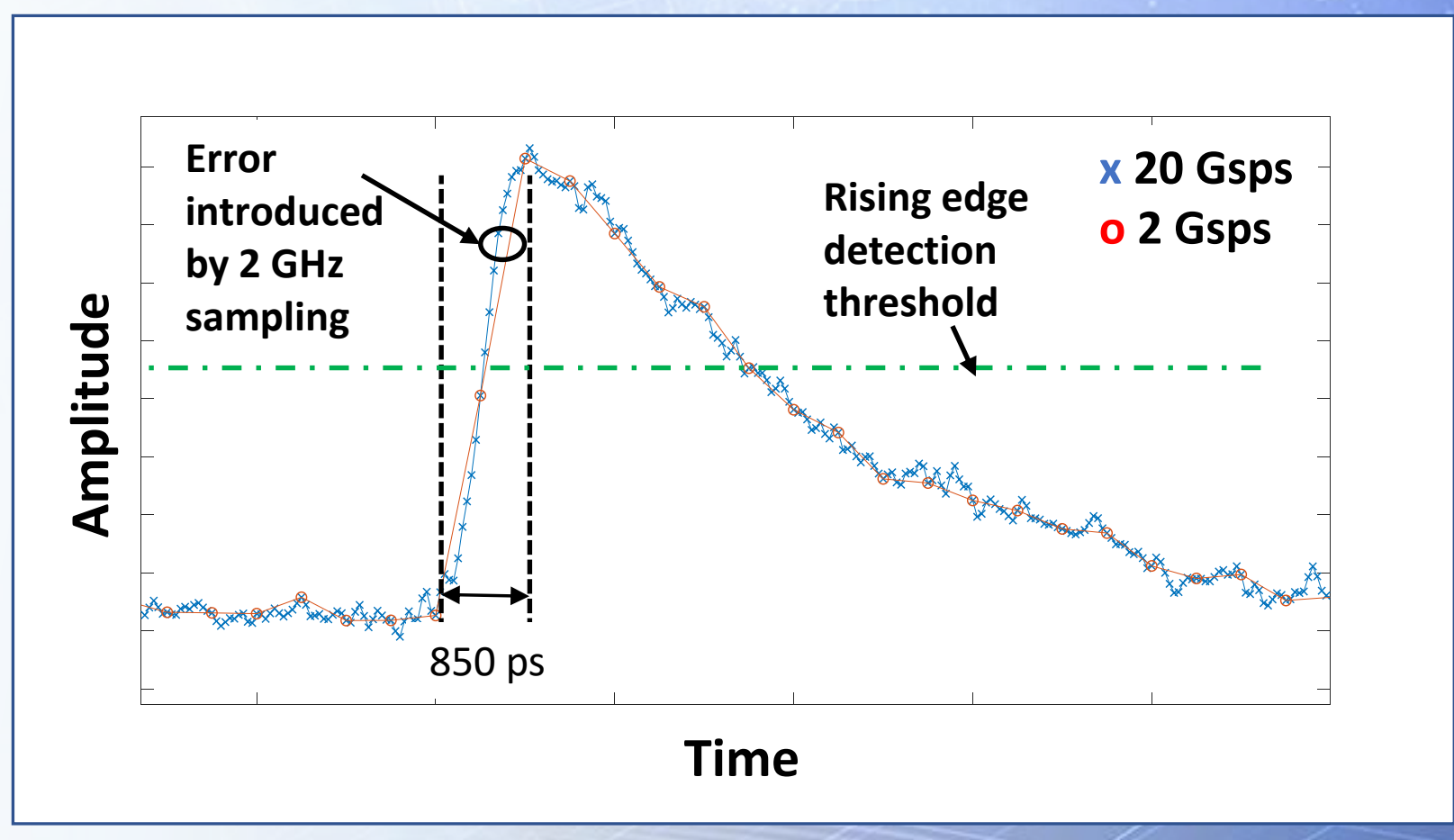

CCSDS High Photon Efficiency Downlink Receive Waveform

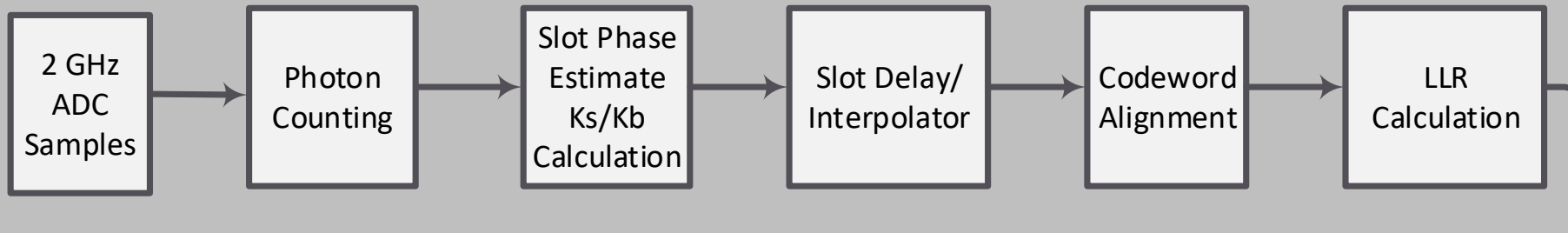

SCPPM Decoder (BCJR Algorithm)

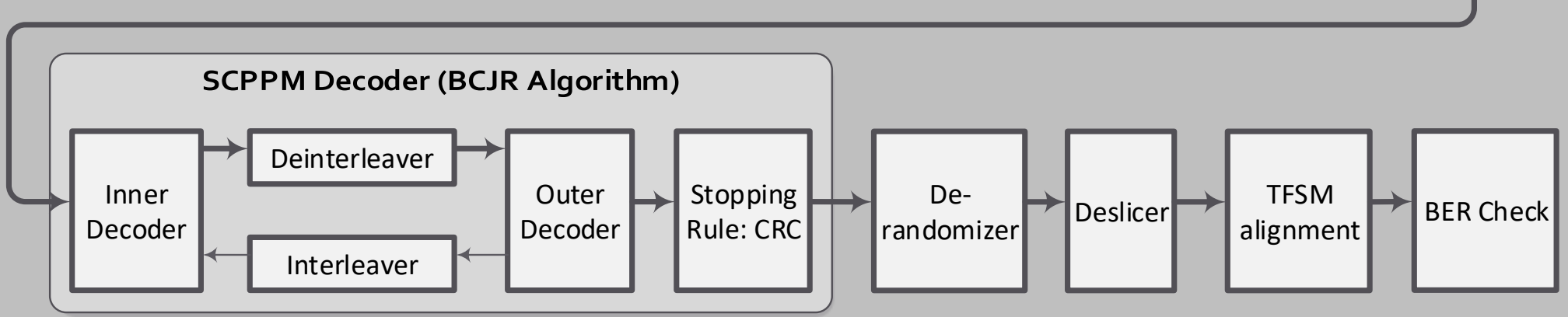




\section{System Simulations}

\section{Description:}

- Matlab model of the CCSDS Optical Communications Coding and Synchronization telemetry link (downlink)

- Transmit waveform

- Floating point receive waveform has iterative decoding using the BCJR algorithm

- Simulations modeled key system parameters including:

- Number of detectors

- Detector blocking

- Jitter

- Background noise photons/slot (Kb)

- Signal photons/signal slot (Ks)

- Performance metrics:

- Bit error rate curves generated for fixed background noise 


\section{System Simulation Results - Capacity and Baseline}

- PPM-32

- Code rate $1 / 3$

- $\mathrm{Kb}=0.0001$ background photons/slot

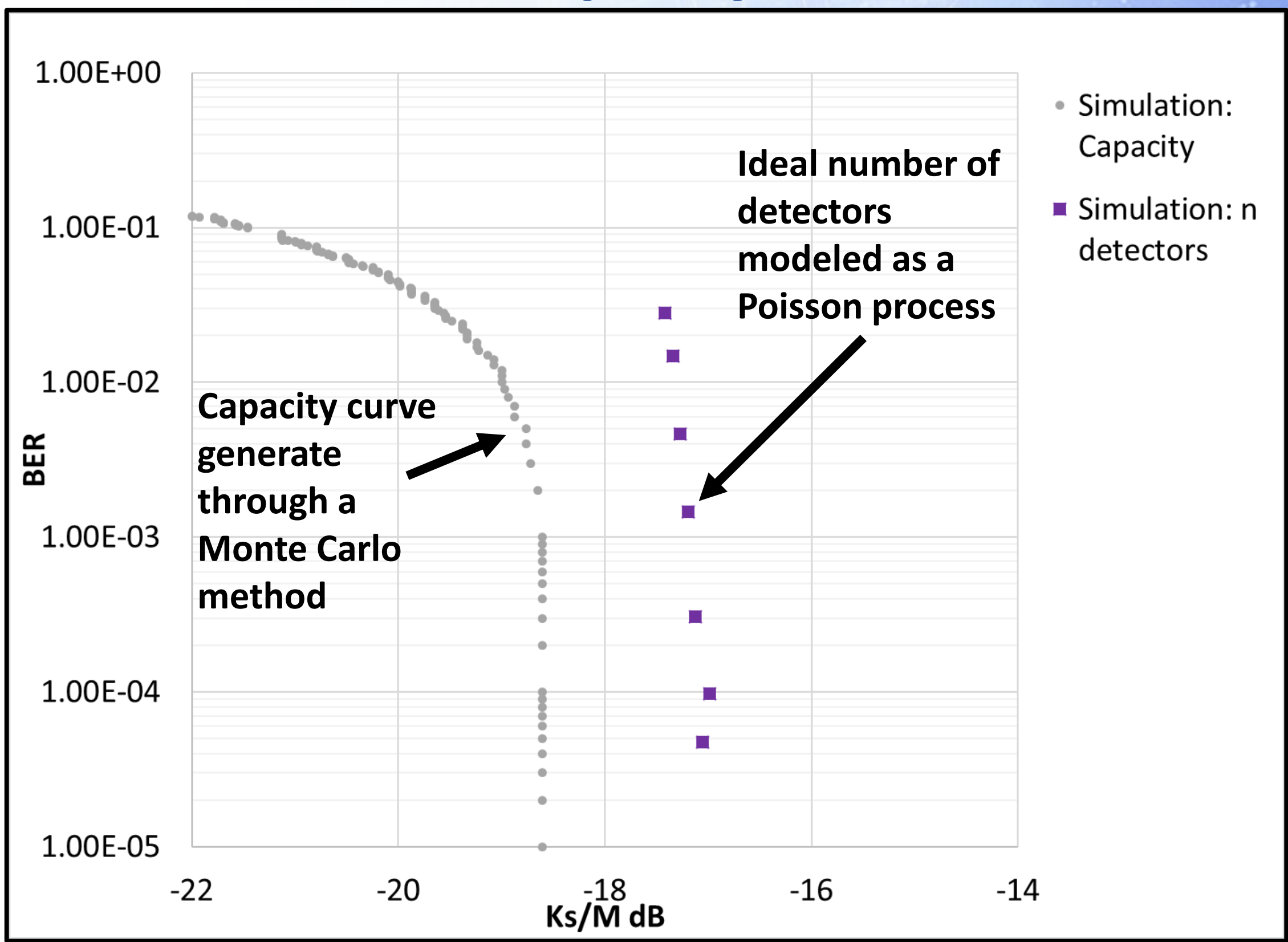

Simulation:

Simulation: $\mathrm{n}$ detectors 


\section{System Simulation Results - 1 Detector}

- PPM-32

- Code rate $1 / 3$

- $\mathrm{Kb}=0.0001$ background photons/slot

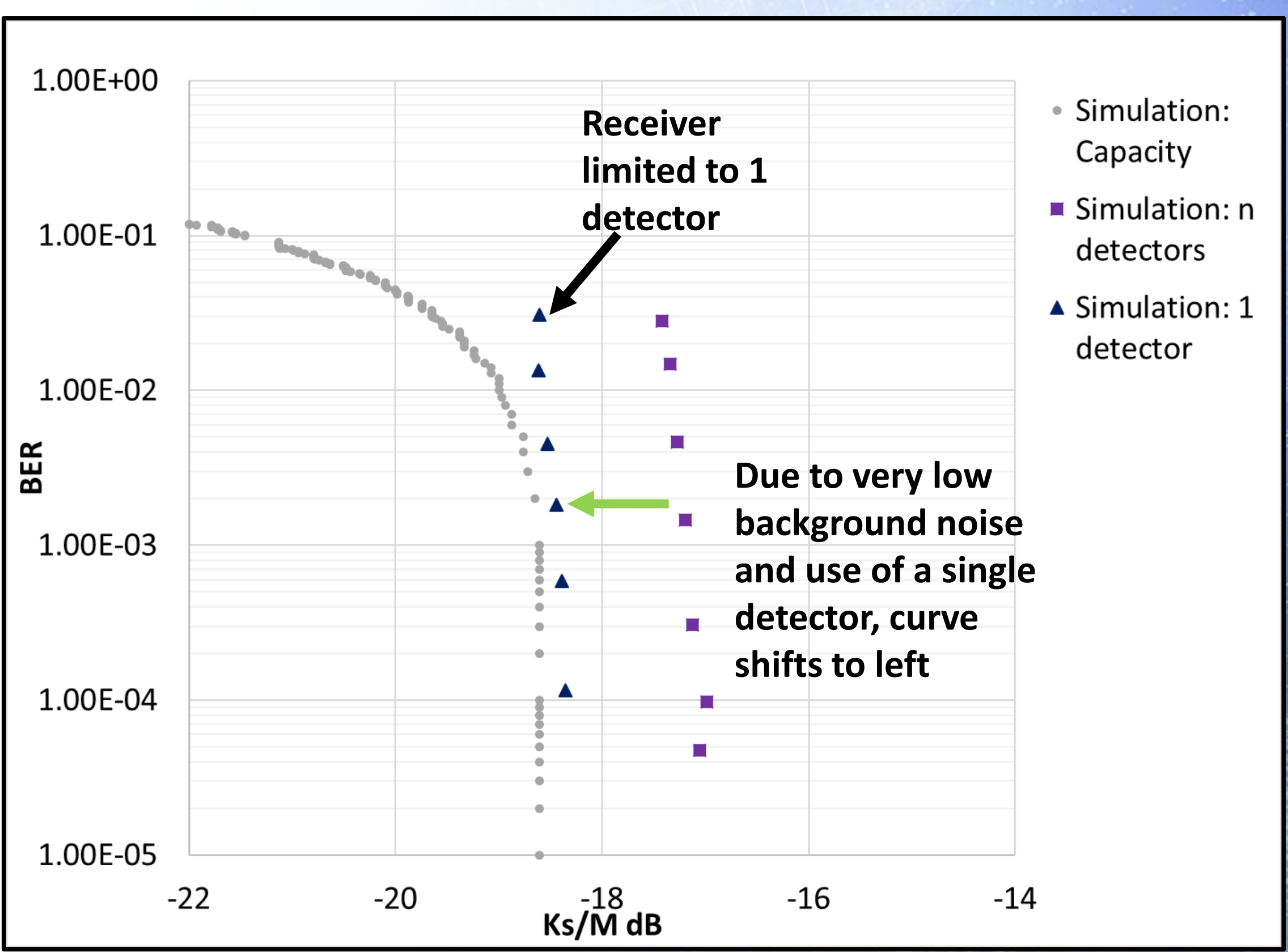

Simulation:

Simulation: $\mathrm{n}$

Simulation: 1

detector 


\section{System Simulation Results -1 Detector +20 ns Blocking}

- PPM-32

- Code rate $1 / 3$

- 1 ns slot width

- Guard band: 8 slots (25\%)

- 40 Mbps data rate

- $\mathrm{Kb}=0.0001$ background photons/slot

No significant change in performance compared to no blocking due to pulse repetition rate of waveform selected

1

- Simulation: Capacity

- Simulation: $\mathrm{n}$ detectors

ه Simulation: 1 detector

- Simulation: 1 detector; 20 ns blocking

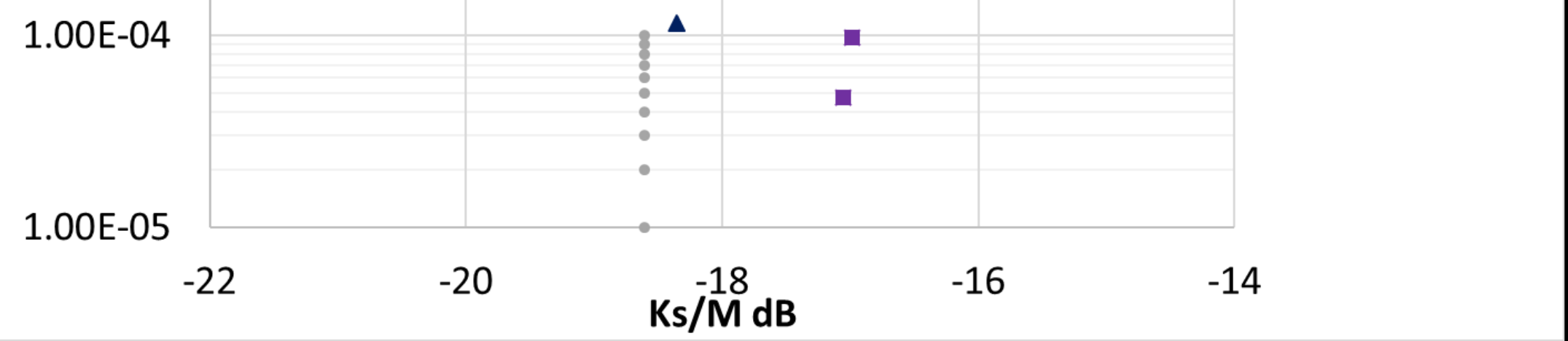




\section{System Simulation Results - Detector Jitter}

- PPM-32

- Code rate $1 / 3$

- 1 ns slot width

- Guard band: 8 slots (25\%)

- 40 Mbps data rate

- $\mathrm{Kb}=0.0001$ background photons/slot

60 ps RMS jitter: $\rightarrow 0.6 \mathrm{~dB}$ loss 80 ps RMS jitter: $\rightarrow 0.8 \mathrm{~dB}$ loss

3

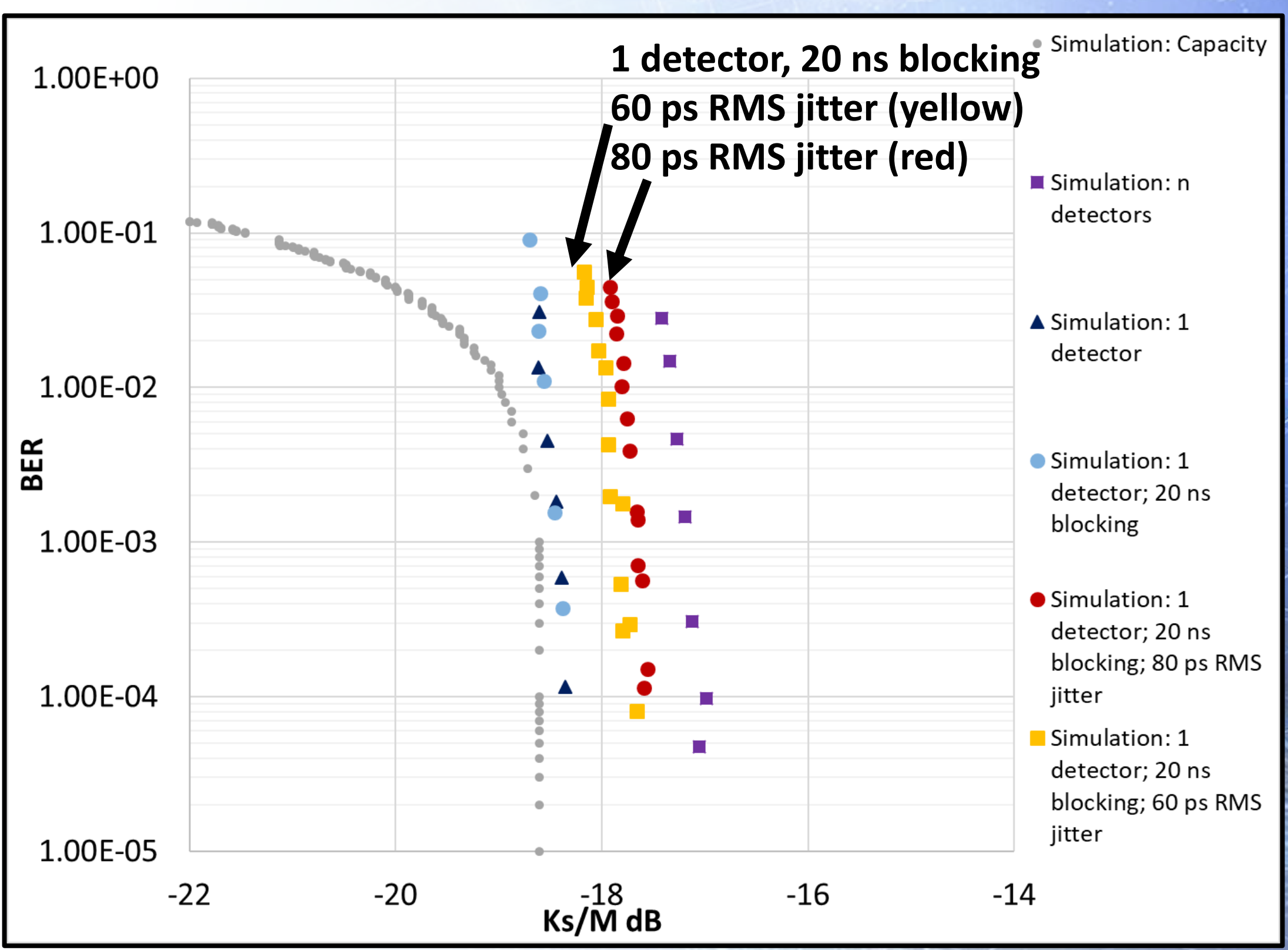
60 ps RMS jitter (yellow) 


\section{Test bed System Testing Results - Channel 1}

- PPM-32

- Code rate $1 / 3$

- 1 ns slot width

- Guard band: 8 slots (25\%)

- 40 Mbps data rate

- $\mathrm{Kb} \approx 0.0001$ background photons/slot

Channel 1 (61 ps RMS jitter) matches simulation of $60 \mathrm{ps}$

RMS jitter

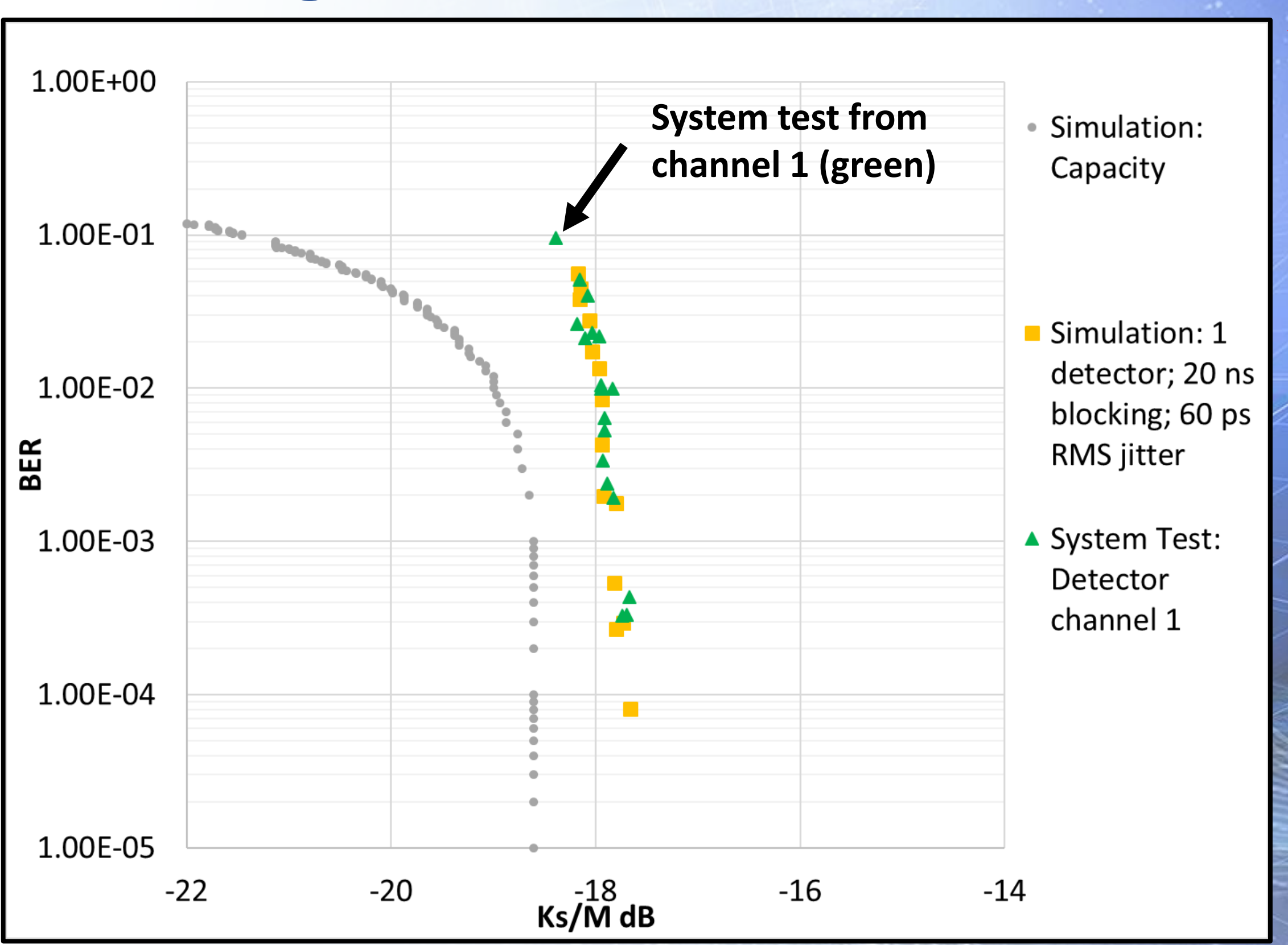

Simulation:

Capacity

Simulation: 1 detector; 20 ns blocking; 60 ps RMS jitter

System Test: Detector channel 1 


\section{Test bed System Testing Results - Channel 2}

- PPM-32

- Code rate $1 / 3$

- 1 ns slot width

- Guard band: 8 slots (25\%)

- 40 Mbps data rate

- $\mathrm{Kb} \approx 0.0001$ background photons/slot

Channel 2 (68 ps RMS jitter) matches simulation of $80 \mathrm{ps}$ RMS jitter

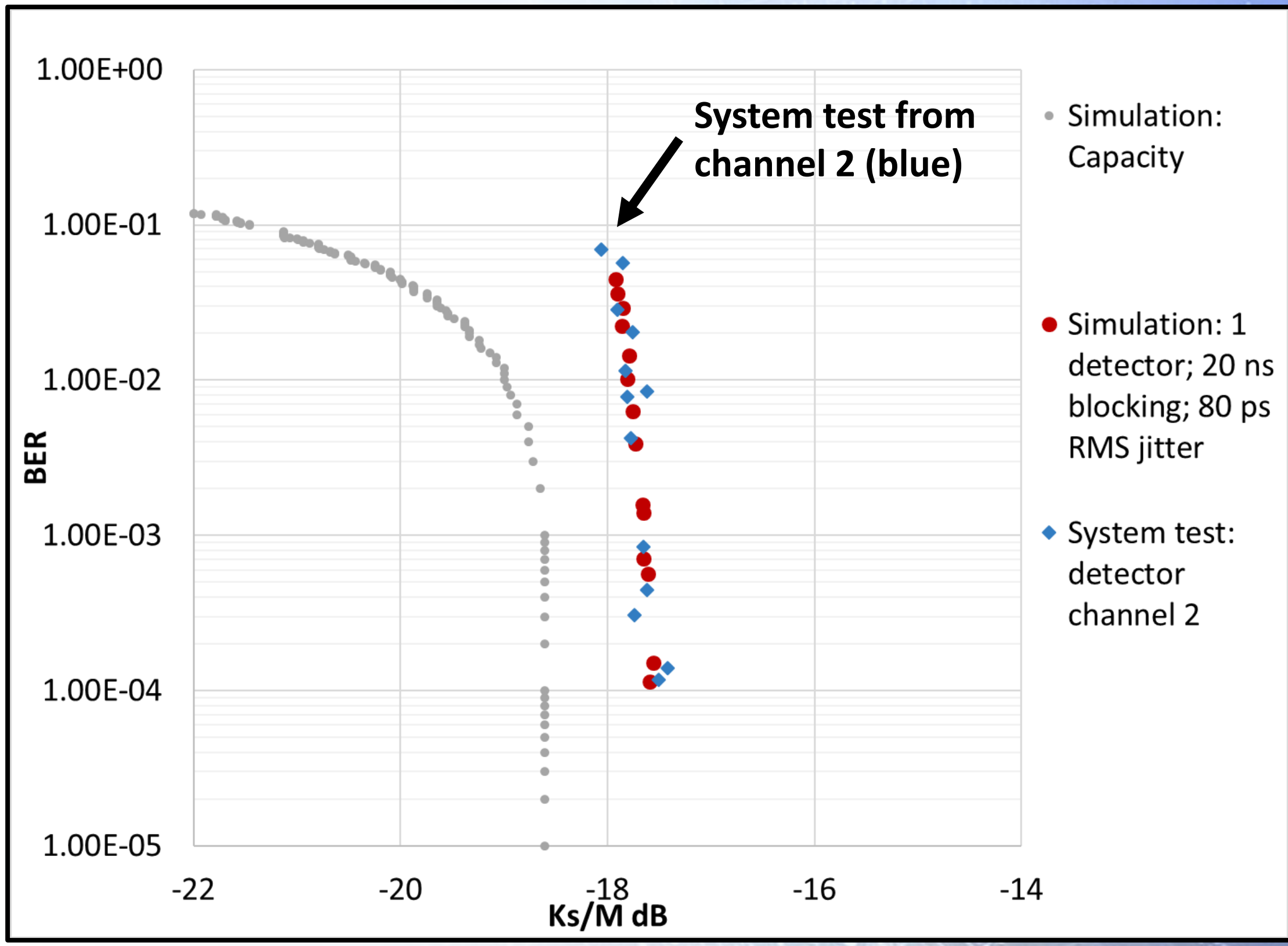

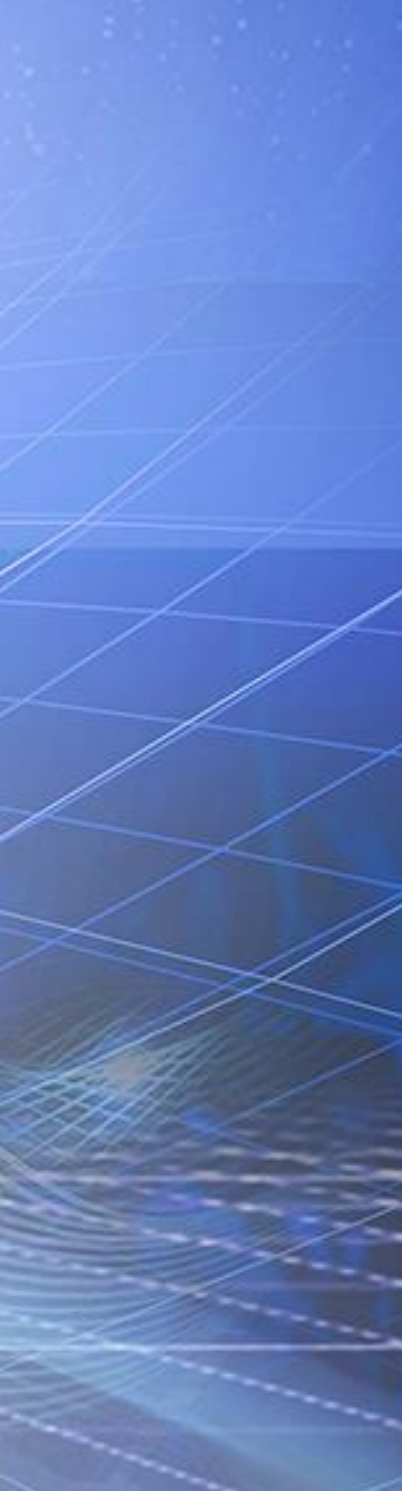




\section{Summary}

- A photon counting optical communications system test bed was designed and characterized

- Key parameters of the system were modeled in simulation including:

- Detector blocking

- Detector jitter

- Detector pulse rise time

- Background noise

- BER curve results from the system test bed align with simulation results

$\rightarrow$ Sources of loss in the system have been accurately characterized

$\rightarrow$ Model can be used to predict performance of other waveforms 


\section{Acknowledgements}

This work was funded by the Space Communications and Navigation Program at NASA. 


\section{Thank You!}

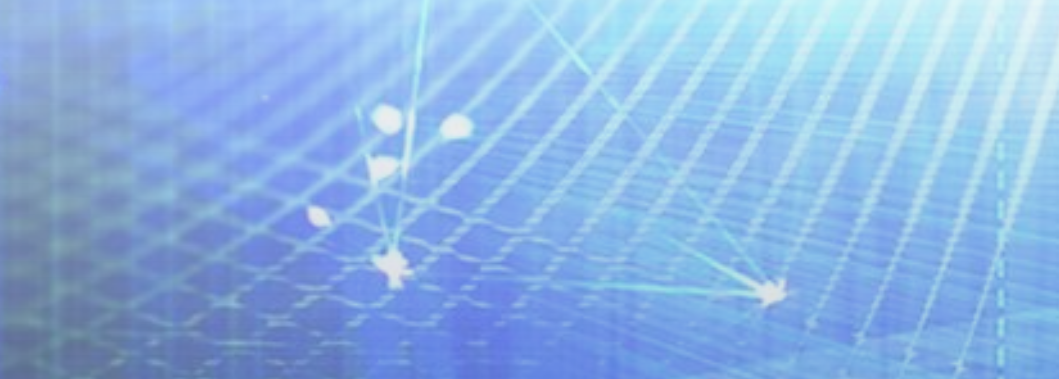

$\frac{0}{4}+1=$ 